\title{
Initial smooth topology for a family of mappings
}

\author{
Kerim Bekar
}

Department of Mathematics, Faculty of Sciences and Arts, Giresun University, Giresun, Turkey

Received: 23 January 2019, Accepted: 6 February 2019

Published online: 26 December 2019.

\begin{abstract}
We consider initial smooth topology for a family of mappings. It is shown that the Šostak's initial smooth topology for family of functions is wrong. In this paper by using K.Chattopadhyay and R.Hazra 's result this mistake has been corrected.
\end{abstract}

Keywords: Smooth topology, initial smooth topology.

\section{Introduction}

In [4], A. Šostak defined the initial smooth topology for a family of mappings. In this paper we show that it is indeed false, and with the help of K.Chattopadhyay and R.Hazra 's result in [2], it may be corrected.

Firstly, let us recall some necessary notions from the theory of smooth topology.

Definition 1. Let $(X, \tau)$ be a smooth topological space and $r \in(0,1]$. $r$ cut of smooth topology $\tau$ is defined as

$$
\tau_{r}:=\left\{\mu \in I^{X}: \tau(\mu) \geq r\right\}
$$

Definition 2. [5] A fuzzy topological space is a pair $(X, T)$, where $X$ is a non-empty set and $T \subset I^{X},(I=[0,1])$ satisfying the following properties:

(1) $0,1 \in T$

(2) $\mu \cap v \in T, \forall \mu, v \in T$

(3) $\bigcup_{i \in \triangle} \mu_{i} \in T, \forall\left\{\mu_{i}: i \in \triangle\right\} \subset T$

Definition 3. [3] A smooth topological space is a pair $(X, \tau)$, where $X$ is a non-empty set and $\tau: I^{X} \longrightarrow I(I=[0,1])$ is a mapping satisfying the following properties:

(O1) $\tau(0)=\tau(1)=1_{I}$

(O2) $\forall \mu, v \in I^{X}, \tau(\mu \cap v) \geq \tau(\mu) \wedge(v)$

(O3) $\forall \triangle, \tau\left(\bigcup_{i \in \triangle} \mu_{i}\right) \geq \bigwedge_{i \in \triangle} \tau\left(\mu_{i}\right)$.

Note that, sometimes a smooth topology is called gradation of openness. Moreover, it is easy to see that if $\tau$ is crisp (i.e. $\left.\tau\left(I^{X}\right) \subset\{0,1\}\right)$, this definition coincides with the known definition of fuzzy topology [1].

Definition 4. [3] A map $f: X \rightarrow Y$ is smooth continuous with respect to the smooth topologies $\tau_{1}$ and $\tau_{2}$ respectively, if for every $\mu \in I^{Y}$ we have

$$
\tau_{1}\left(f^{-1}(\mu)\right) \geq \tau_{2}(\mu)
$$

\footnotetext{
* Corresponding author e-mail: kebekar@gmail.com
} 
where $f^{-1}(\mu)$ is defined by $f^{-1}(\mu)(x)=\mu(f(x))$.

Proposition 1. [3] Let $\left\{T_{\alpha}: \alpha \in(0,1]\right\}$ be a family of fuzzy topologies on $X$ such that $\alpha_{1}>\alpha_{2}$ implies $T_{\alpha_{1}}<T_{\alpha_{2}}$.

$$
\tau(\mu):=\bigvee\left\{\alpha: \mu \in T_{\alpha}\right\}
$$

Then $\tau$ is a smooth topology on $X$.

Definition 5. [5] Let $\tau_{1}$ and $\tau_{2}$ be smooth topology on X.If $\tau_{1}>\tau_{2}$ then $\tau_{2}$ is the weakest than $\tau_{1}$.

Definition 6. [4] Let $X$ be a set, $(Y, \tau)$ be a smooth topological space and $f: X \rightarrow Y$ be a mapping. The weakest smooth topology $\delta$ on $X$ such that the mapping

$$
f:(X, \delta) \rightarrow(Y, \tau)
$$

is smooth continuous, is called the initial smooth topology for a mapping.

To construct such a smooth topology consider the set $M:=\left\{f^{-1}(v): v \in I^{Y}\right\}$ of smooth subsets of $X$. For a given $\mu \in M$ let

$$
P_{\mu}:=\left\{v \in I^{Y}: \mu=f^{-1}(v)\right\}
$$

and define $\delta(\mu):=\bigvee\left\{\tau(v): v \in P_{\mu}\right\}$

Definition 6 can be generalized for a family of mappings as follows.

Definition 7. [4] Let $X$ be a set, $\left\{\left(Y_{i}, \tau_{i}\right): i \in \triangle\right\}$ be a family of smooth topological spaces and for each $i \in \triangle$

$$
f_{i}: X \longrightarrow\left(Y_{i}, \tau_{i}\right)
$$

be a mapping. The weakest smooth topology $\tau$ on $X$ such that the mapping

$$
f_{i}:(X, \tau) \longrightarrow\left(Y_{i}, \tau_{i}\right)
$$

is smooth continuous for each $i \in \triangle$, is called initial smooth topology for a family of mappings.

Let us to remind the Šostak's definition of initial smooth topology for a family of mappings (see [4, (3.2)]). For each $i \in \triangle$, let $\delta_{i}: I^{X} \rightarrow I$ be the initial smooth topology on $X$ for $f_{i}$, i.e. it is the weakest smooth topology on $X$ such that the mapping

$$
f_{i}:\left(X, \delta_{i}\right) \rightarrow\left(Y_{i}, \tau_{i}\right)
$$

is smooth continuous. Let the mapping $\tau: I^{X} \rightarrow I$ be defined by the equality

$$
\tau(\mu)=\bigwedge\left\{\delta_{i}(\mu): i \in \Delta\right\}
$$

where $\mu \in I^{X}$. Šstak in [4] asserted that $\tau$ is an initial smooth topology for a family $\left(f_{i}\right)$, i.e. $\tau$ is the weakest smooth topology on $X$ such that the mapping $f_{i}$ is smooth continuous for each $i \in \triangle$.

\section{General properties}

We assert that the assertion of Šstak of before is not true. General speaking, for any $i \in \triangle$ there is no need for $f_{i}$ to be smooth continuous relative to the smooth topology $\tau$, as shown by the following example. 
Example 1. Let $Y$ be a set, $\tau_{1}$ and $\tau_{2}$ be the two smooth topology on $Y$, and for $X=Y$ and some fuzzy set $\mu_{o} \in I^{X}$, $\tau_{1}\left(\mu_{o}\right)=1$ and $\tau_{2}\left(\mu_{o}\right)=0$. Let

$$
i d_{1}: X \rightarrow\left(Y, \tau_{1}\right)
$$

and $i d_{2}: X \rightarrow\left(Y, \tau_{2}\right)$ be the identity mappings. Assume that $\delta_{1}$ and $\delta_{2}$ are the initial smooth topologies corresponding to the mappings $i d_{1}$ and $i d_{2}$, respectively; and $\delta$ is the initial smooth topology in the sense of Šostak, for a family $\left\{i d_{j}\right\}_{j=1}^{2}$. We show that, for example the mapping $i d_{1}$ isn't smooth continuous relative to $\delta$.

Indeed, it is clear that $\delta_{1}=\tau_{1}$ and $\delta_{2}=\tau_{2}$. According to Šostak for $\mu \in I^{X}, \delta(\mu)=\bigwedge_{i} \delta_{i}(\mu)$. Since for $\mu_{o} \in I^{X}$

$$
\begin{aligned}
\delta\left(i d_{1}^{-1}\left(\mu_{o}\right)\right) & =\delta\left(\mu_{o}\right) \\
& =\delta_{1}\left(\mu_{o}\right) \wedge \delta_{2}\left(\mu_{o}\right) \\
& =\tau_{1}\left(\mu_{o}\right) \wedge \tau_{2}\left(\mu_{o}\right) \\
& =1 \wedge 0=0 \nsupseteq 1=\tau_{1}\left(\mu_{o}\right),
\end{aligned}
$$

then

$$
\delta\left(i d_{1}^{-1}\left(\mu_{o}\right)\right) \nsupseteq \tau_{1}\left(\mu_{o}\right) .
$$

Hence the mapping $i d_{1}$ isn't smooth continuous relative to $\delta$.

Essentially the right construction of the initial smooth topology for a family mappings is included in [2, Proposition 5.3]. Let us recall this Proposition.

Theorem 1. [2] Let $X$ be a set, $\left\{\left(X_{i}, \tau_{i}^{\prime}\right): i \in \triangle\right\}$ be a family of the smooth topological spaces and for $i \in \triangle$

$$
f_{i}: X \rightarrow X_{i}
$$

be a mapping. Then there exists a smooth topology $\tau$ on $X$ such that the following conditions hold:

(i) for each $i \in \triangle, f_{i}:(X, \tau) \rightarrow\left(X_{i}, \tau_{i}^{\prime}\right)$ is smooth continuous;

(ii) if $\left(Z, \tau^{\prime \prime}\right)$ is a smooth topological space then $g:\left(Z, \tau^{\prime \prime}\right) \rightarrow(X, \tau)$ is smooth continuous iff

$$
f_{i} \circ g:\left(Z, \tau^{\prime \prime}\right) \rightarrow\left(X_{i}, \tau_{i}^{\prime}\right)
$$

is smooth continuous for each $i \in \triangle$.

In the proof of Theorem 1 (i.e. of the Proposition 5.3, [2]) the smooth topology $\tau$ on $X$ was defined in [2] as the follows: For each $i \in \triangle$ and $\alpha \in(0,1]$, define

$$
\top_{i, \alpha}:=\left\{f_{i}^{-1}(\mu): \mu \in\left(\tau_{i}^{\prime}\right)_{\alpha}\right\}
$$

Recall that

$$
\left(\tau_{i}^{\prime}\right)_{\alpha}=\left\{\mu \in I^{X}: \tau_{i}^{\prime}(\mu) \geq \alpha\right\}
$$

is the $\alpha$-level fuzzy topology on $X_{i}$ and $\left\{T_{i, \alpha}: \alpha \in(0,1]\right\}$ is a descending chain of fuzzy topologies on $X$. For each $\alpha \in(0,1]$, define $\mathscr{S}_{\alpha}:=\bigcup_{i} \top_{i, \alpha}$ and $\top_{\alpha}$ be the fuzzy topology on $X$ generated by $\mathscr{S}_{\alpha}$ as a subbase. It can be verified that $\left\{T_{\alpha}: \alpha \in(0,1]\right\}$ is a descending chain of fuzzy topologies on $X$. Moreover we have a gradation of openness, $\tau$, on $X$ associated to $\left\{\top_{\alpha}: \alpha \in(0,1]\right\}$, where $\tau(\lambda)=\bigvee\left\{\alpha \in(0,1]: \lambda \in \top_{\alpha}\right\}$.

By Theorem 1 (i) for each $i \in \triangle, f_{i}:(X, \tau) \rightarrow\left(X_{i}, \tau_{i}\right)$ is smooth continuous. By (ii), $\tau$ is the weakest smooth topology on 
$X$ such that the mapping $f_{i}$ is smooth continuous for each $i \in \triangle$.

Indeed, if $\tau_{o}$ is an arbitrary smooth topology on $X$ such that $f_{i}:\left(X, \tau_{o}\right) \rightarrow\left(X_{i}, \tau_{i}\right)$ is smooth continuous for each $i \in \triangle$, then by Theorem 1 (ii), id $:\left(X, \tau_{0}\right) \rightarrow(X, \tau)$ must be smooth continuous, since for each

$$
f_{i}=f_{i} \circ i d:\left(X, \tau_{0}\right) \rightarrow\left(X_{i}, \tau_{i}\right)
$$

is smooth continuous, where $i d$ is the identity mapping. Hence for each $\mu \in I^{X}, \tau_{o}\left(i d^{-1}(\mu)\right) \geq \tau(\mu)$, i.e. $\tau_{o}(\mu) \geq \tau(\mu)$. Therefore $\tau_{o} \geq \tau$. The above proof can be given in more detail as below. Let $\left\{\left(X_{i}, \tau_{i}^{\prime}\right): i \in \triangle\right\}$ be a family of smooth topological spaces and $\left\{f_{i}: X \rightarrow X_{i}: i \in \triangle\right\}$ be a family of functions. Let

$$
\left(\tau_{i}^{\prime}\right)_{r}=\left\{\lambda \in I^{X_{i}}: \tau_{i}^{\prime}(\lambda) \geq r\right\}, \forall i \in \triangle \text { and } \forall r \in(0,1]
$$

and

$$
T_{i, r}:=\left\{f_{i}^{-1}(\lambda): \lambda \in\left(\tau_{i}^{\prime}\right)_{r}\right\}, \forall i \in \triangle \text { and } \forall r \in(0,1]
$$

In addition, let's define as

$$
\mathscr{S}_{r}:=\bigcup_{i \in \triangle} T_{i, r}, \forall r \in(0,1]
$$

Let $T_{r}$ be the fuzzy topology which accepts $\mathscr{S}_{r}$ a subbase on $X$.

$$
\tau(\mu):=\bigvee\left\{r \in(0,1]: \mu \in T_{r}\right\} \text { for } \mu \in I^{X}
$$

is a smooth topology on $X$ [3]. Moreover, $\tau$ is the weakest topology that makes smooth continuous to each function

$$
f_{i}:(X, \tau) \longrightarrow\left(X_{i}, \tau_{i}\right)
$$

on $X$. Now, let's prove it.

Let $\tau^{\prime}$ be any smooth topology that makes smooth continuous to functions

$$
f_{i}:\left(X, \tau^{\prime}\right) \longrightarrow\left(X_{i}, \tau_{i}\right)
$$

on $X$ and be $\mu \in I^{X}$. Let

$$
s:=\tau(\mu)=\bigvee\left\{r \in(0,1]: \mu \in T_{r}\right\}
$$

There exists at least $r \in(0,1]$ for each $\varepsilon>0$ such that $r>s-\varepsilon$ and $\mu \in T_{r}$. Since $\mu \in T_{r}$ ve $\mathscr{S}_{r}$ is subbase,

$$
\exists \mu_{\gamma, k} \in \mathscr{S}_{r}, \quad(\gamma \in \Lambda \text { and } k \in\{1,2, \ldots, n\}) \text { such that } \mu=\bigcup_{\gamma \in \Lambda k=1}^{n} \bigcap_{\gamma, k} .
$$

For every $\mu_{\gamma, k} \in \mathscr{S}_{r}$, there exists at least $i_{\gamma, k} \in \triangle$ such that $\mu_{\gamma, k} \in T_{i_{\gamma, k}, r}$. From the definiton of $T_{i_{\gamma, k}, r}$,

$$
\exists \lambda_{\gamma, k} \in\left(\tau_{i_{\gamma, k}}^{\prime}\right)_{r} \text { such that } \mu_{\gamma, k}:=f_{i_{\gamma, k}}^{-1}\left(\lambda_{\gamma, k}\right), \forall \gamma \in \Lambda, \forall k \in\{1,2, \ldots, n\} \text {. }
$$

Therefore,

$$
\tau_{i_{\gamma, k}^{\prime}}^{\prime}\left(\lambda_{\gamma, k}\right) \geq r, \forall \gamma \in \Lambda \text { and } \forall k \in\{1,2, \ldots, n\}
$$


From the hypothesis, since $f_{i}:\left(X, \tau^{\prime}\right) \longrightarrow\left(X_{i,} \tau_{i}\right)$ is smooth continuous for every $i \in \triangle, f_{i_{\gamma, k}}:\left(X, \tau^{\prime}\right) \longrightarrow\left(X_{i_{\gamma, k},} \tau_{i_{\gamma, k}}\right)$ is smooth continuous for every $\gamma \in \Lambda$ and every $k \in\{1,2, \ldots, n\}$ and

$$
\tau^{\prime}\left(f_{i_{\gamma, k}}^{-1}\left(\lambda_{\gamma, k}\right)\right) \geq \tau_{i_{\gamma, k}}^{\prime}\left(\lambda_{\gamma, k}\right) \geq r
$$

From here,

$$
\tau^{\prime}\left(f_{j_{\gamma, k}}^{-1}\left(\lambda_{\gamma, k}\right)\right) \geq r \text { for } \forall \gamma \in \Lambda \text { and } \forall k \in\{1,2, \ldots, n\}
$$

From (2) and (4), $\tau^{\prime}\left(\mu_{\gamma, k}\right) \geq r$ for every $\gamma \in \Lambda$ and every $k \in\{1,2, \ldots, n\}$. Since $\tau^{\prime}$ is the smooth topology,

$$
\tau^{\prime}\left(\bigcup_{\gamma \in \Lambda} \bigcap_{k=1}^{n} \mu_{\gamma, k}\right) \geq r
$$

From (1), $\tau^{\prime}(\mu) \geq r>s-\varepsilon$. Since $\varepsilon>0$ is arbitrary, $\tau^{\prime}(\mu) \geq s=\tau(\mu)$. That is, $\tau^{\prime}(\mu) \geq \tau(\mu)$. Since $\mu \in I^{X}$ is arbitrary, $\tau^{\prime} \geq \tau$. Therefore, since $\tau^{\prime}$ is also arbitrary, $\tau$ is the weakest topology that makes smooth continuous to every function $f_{i}:(X, \tau) \longrightarrow\left(X_{i}, \tau_{i}\right)$ on $X$.

\section{Conclusion}

In this paper, we considered initial smooth topology for a family of mappings. We showed that the Šostak's initial smooth topology for family of functions is wrong. After that, we gave the correct concept.

\section{Acknowledgements}

The author would like to express his gratitude to Prof. Ali Bülbül for usefull discassions. This paper was produced from the author's master thesis [6].

\section{Competing interests}

The authors declare that they have no competing interests.

\section{Authors' contributions}

All authors have contributed to all parts of the article. All authors read and approved the final manuscript.

\section{References}

[1] Chang, C., Fuzzy topological spaces, J. Math. Anal. Appl. 24 (1968) 182-190.

[2] Chattopadhyay, K., Hazra, R. and Samanta, S., Gradation of openness:fuzzy topology, Fuzzy Sets and Systems 49 (1992) $237-242$.

[3] Ramadan, R., Smooth topological spaces, Fuzzy Sets and Systems 48 (1992) 371-375.

[4] Šostak, A., On a Fuzzy Topological Structure, Rend. Circ. Matem. Palermo Ser. II, 11 (1985) 89-103.

[5] Šostak,A., On Some Modifications of Fuzzy Topology, Mat. Vesnik, 41 (1989) 51-64.

[6] Bekar K., Smoot h topolok uzaylar. Karadeniz Teknical University, Fen Bilimleri Enstitusu, Matematik Anabilim Dali, Yuksek Lisans Tezi, Ocak 1999.Trabzon. 